\section{CALIDAD DE FRUTOS DE TOMATES SILVESTRES (Lycopersicon esculentum var. cerasiforme Dunal) DE OAXACA, MÉXICO}

\section{FRUIT QUALITY OF WILD TOMATOES (Lycopersicon esculentum var. cerasiforme Dunal) FROM OAXACA, MÉXICO}

\author{
Andrés U. Crisanto-Juárez ${ }^{1}$, Araceli M. Vera- \\ Guzmán $^{2}$, José L. Chávez-Servia ${ }^{2 *}$ y José C. \\ Carrillo-Rodríguez ${ }^{3}$
}

\begin{abstract}
${ }^{1}$ Instituto Tecnológico de Oaxaca, Av. V. Bravo Ahuja \# 125. 68030, Oaxaca, México. ${ }^{2}$ Centro Interdiciplinario de Investigacion para el Desarrollo Integral-Instituto Politécnico Nacional Unidad Oaxaca, Hornos \# 1003, Santa Cruz Xoxocotlán. 71230, Oaxaca, México. ${ }^{3}$ Instituto Tecnológico del Valle de Oaxaca, 71230, Ex-Hacienda Nazareno. Santa Cruz Xoxocotlán, Oaxaca, México.
\end{abstract}

* Autor para correspondencia (jchavezservia@yahoo.com)

\section{RESUMEN}

México posee diversos acervos genéticos de tomate (Lycopersicon esculentum Mill.) que fueron generados durante largos procesos de domesticación y diversificación, pero poco documentados en cuanto a calidad de frutos. Para evaluar la calidad física, química y nutricional de frutos de tomate silvestre (L. esculentum var. cerasiforme Dunal), se hizo una colecta de 17 muestras en ocho municipios del estado de Oaxaca, México. Las semillas de las muestras se sembraron, bajo un diseño de bloques al azar con tres repeticiones, en invernadero durante el ciclo primavera-verano 2009. En cada parcela experimental se tomó una muestra de frutos maduros (rojos), firmes y sanos, en la que se evaluaron los contenidos de licopeno, vitamina $\mathrm{C}$, sólidos solubles, $\mathrm{pH}$, azúcares reductores, acidez titulable, y las coordenadas cromáticas $L^{*}, a^{*}$ y b*. Mediante un análisis de componentes principales se determinó que los contenidos de licopeno, sólidos solubles, pH, azúcares reductores, acidez titulable, y los parámetros de color, fueron las variables más importantes para describir la variabilidad evaluada. Un análisis de conglomerados reveló la presencia de tres grupos entre las colectas evaluadas. Se presentaron diferencias $(P<0.05)$ entre grupos y entre colectas en todas las variables. Las colectas $L-$ 063 y L-073, ambas de San Blas Atempa, sobresalieron en licopeno en base húmeda con 26.8 y $28.2 \mathrm{mg} 100 \mathrm{~g}^{-1}$, $\mathrm{y}$ en base seca con 368.1 y $343.9 \mathrm{mg} 100 \mathrm{~g}^{-1}$, respectivamente. En vitamina $\mathrm{C}$ sobresalieron las colectas L-074 de Santiago Nuyoó, L-076, L-077, L-078 y L-079 de San Blas Atempa, y L-080 de Nejapa de Madero, con más de $15 \mathrm{mg}$ $100 \mathrm{~g}^{-1}$. El índice de color $\left(\mathrm{a}^{*} / \mathrm{b}^{*}\right)$ correlacionó positivamente con todos los caracteres químicos y nutricionales, excepto con $\mathbf{p H}$.

Palabras clave: Lycopersicon esculentum var. cerasiforme, antioxidantes, licopeno, postcosecha.

\section{SUMMARY}

México has diverse gene pools of tomato (Lycopersicon esculentum Mill.) that have resulted from extended domestication and diversification processes, but are poorly documented in terms of fruit quality. To evaluate the physical, chemical and nutritional quality of wild tomato fruits (L. esculentum var. cerasiforme Dunal), 17 fruit samples were collected from eight municipalities in Oaxaca State, México. Seeds from each sample were planted, following a randomized block design with three replications, under greenhouse conditions during Spring-Summer 2009. From each experimental plot, a sample of fruits (all fully red, firm and healthy) was analyzed for content of lycopene, vitamin $\mathrm{C}$, soluble solids, $\mathrm{pH}$, sugars, titratable acidity, and chromatic coordinates $\mathrm{L}^{*}, \mathrm{a}^{*}$ and $\mathrm{b}^{*}$. According to a principal components analysis, the content of lycopene, soluble solids, $\mathrm{pH}$, sugars, titratable acidity and color parameters were the most important traits for describing fruit variability. The 17 accessions were classified into three groups by a cluster analysis. Differences $(P<\mathbf{0 . 0 5})$ were found among clusters and among accessions for all variables. Accessions L-063 and L-073, both from San Blas Atempa, stood out for their lycopene content on fresh weight (26.8 and $\left.28.2 \mathrm{mg} 100 \mathrm{~g}^{-1}\right)$, as well as on dry weight (368.1 y $343.9 \mathrm{mg} 100 \mathrm{~g}^{-1}$ ). For vitamin $\mathrm{C}$, accessions $\mathrm{L}-074$ from Santiago Nuyoó, L-076, L-077, L-078 and L-079 from San Blas Atempa, and L-080 from Nejapa de Madero, stood out with more

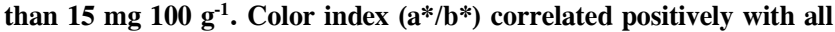
chemical and nutritional traits, except $\mathrm{pH}$.

Index words: Lycopersicon esculentum var. cerasiforme, antioxidants, lycopene, postharvest.

\section{INTRODUCCIÓN}

Una teoría de domesticación del tomate (Lycopersicon esculentum Mill.) indica que tal evento se realizó en México, en tanto que otra señala que fue en Perú, pero tampoco se descarta que tal evento ocurriera en ambos sitios (Rick y Fobes, 1975; Peralta y Spooner, 2007). El origen exacto del tomate cultivado permanece sin resolverse; no obstante, en México la especie continua diversificándose en los trópicos y subtrópicos, donde es conocida como tomatillo (L. esculentum var. cerasiforme Dunal) o con otros nombres locales, como reportó Jenkins (1948), y la evolución continúa (Álvarez-Hernández et $a l .$, 2009). Aunque el tomatillo es muy popular en los Estados de Tabasco, Chiapas, Veracruz, Oaxaca, Guerrero, Puebla, Michoacán, y Jalisco (Lépiz y Rodríguez, 2006), poco se conoce del material silvestre; en términos de su potencial nutricional y características de calidad postcosecha.

Juárez-López et al. (2009) compararon los contenidos de sólidos solubles, acidez titulable, licopeno, $\beta$-caroteno y ácido ascórbico, en siete tipos de tomatillo originarios de Guerrero y Puebla. A nivel nacional sólo se reportan 20 accesiones en el banco de germoplasma de la Universidad Autónoma Chapingo-Centro Regional Oaxaca (Córdoba y Molina, 2006); aunque no se descarta 
que existan otras colecciones del tomate nativo oaxaqueño en diferentes universidades o centros de investigación nacionales y extranjeros. Esto indica, entre otras cosas, la reducida o nula documentación existente acerca del tomate nativo oaxaqueño.

Diversos estudios epidemiológicos han reportado un efecto benéfico del consumo de tomate, porque el licopeno que contiene ayuda a prevenir algunas enfermedades crónicas, como los cánceres de próstata, ovárico, gástrico y pancreático, y enfermedades cardiovasculares (Kavanaugh et al., 2007; Singh y Goyal, 2008). Particularmente, el tomate Cereza ("Cherry") tiende a generar valores nutricionales y antioxidantes más acentuados que los tomates de tipos Saladette y Bola, y así le proporcionan un sabor característico. Raffo et al. (2002) determinaron concentraciones de licopeno de 141 mg $100 \mathrm{~g}^{-1}$ en base seca por cromatografía de líquidos en la variedad tipo "Cherry" "Naomi F1', y George et al. (2004) por espectrofotometría encontraron una variación en licopeno de 3.78 a $6.94 \mathrm{mg} 100 \mathrm{~g}^{-1}$ y de 2.04 a 5.22 $\mathrm{mg} 100 \mathrm{~g}^{-1}$ en base húmeda, en tres variedades tipo "Cherry" y ocho variedades tipo Saladette y Bola, respectivamente; también determinaron patrones similares para el contenido de ácido ascórbico. Debe tomarse en cuenta que los contenidos de ácido ascórbico y licopeno varían con el estado de desarrollo del fruto y con el ambiente de cultivo; al respecto, Slimestad y Verheul (2005) determinaron en la variedad 'Jennita' de tipo "Cherry" una variación inconsistente de 5.65 a 20.2 y de 1.6 a $5.54 \mathrm{mg} 100 \mathrm{~g}^{-1}$ en base húmeda, de ácido ascórbico y licopeno, respectivamente, en 14 cosechas realizadas en diferentes fases de la producción en invernadero. El objetivo del estudio fue evaluar la calidad física, química y nutricional de frutos de tomate de origen silvestre recolectados en distintas regiones del Estado de Oaxaca, México.

\section{MATERIALES Y MÉTODOS}

\section{Material vegetal}

Se colectaron 17 muestras de frutos de tomate de plantas localizadas en solares o traspatios de los agricultores, y en la vegetación cercana a las comunidades y cultivos de maíz (Zea mays L.) en los municipios de Ayotzintepec (L-049 y L-053), San Pedro Ixcatlán (L052), Santa María Guienagati (L-058), San Blas Atempa (L-062，L-063，L-064，L-072，L-073，L-076，L-077, L078 y L-079), Teotitlán de Flores Magón (L-069), Santiago Nuyoó (L-074), Nejapa de Madero (L-080) y Santa María Jacatepec (L-051), Oaxaca. Cada muestra, denominada aquí como colecta, se constituyó por todos los frutos maduros de dos a 10 plantas individuales. La variación altitudinal de las zonas de colecta fue de 30 a $1640 \mathrm{~m}$, todas dentro de las coordenadas $16^{\circ} 20^{\prime}$ y $18^{\circ}$ $09^{\prime} \mathrm{LN}$, y $95^{\circ} 14^{\prime}$ y $97^{\circ} 46^{\prime} \mathrm{LO}$.

Las semillas de los frutos colectados fueron sembradas en invernadero y las plantas manejadas bajo el sistema de fertirrigación, durante el ciclo primavera-verano 2009 (marzo-junio), bajo un diseño experimental de bloques completos al azar con tres repeticiones, en la Ex-Hacienda Nazareno, Santa Cruz, Xoxocotlán, Oaxaca $\left(96^{\circ} 43^{\prime}\right.$ LO, $17^{\circ}$ 04' LN, con altitud de $1519 \mathrm{~m}$ ). Para los análisis de laboratorio, en cada parcela experimental se cosechó, entre el tercero y quinto racimos, una muestra de 200 a $400 \mathrm{~g}$ de frutos, y de la muestra se seleccionaron los frutos completamente rojos (estado de color 6 USDA), firmes al tacto y sanos, criterios basados en Raffo et al. (2002), como se ilustra en la Figura 1.

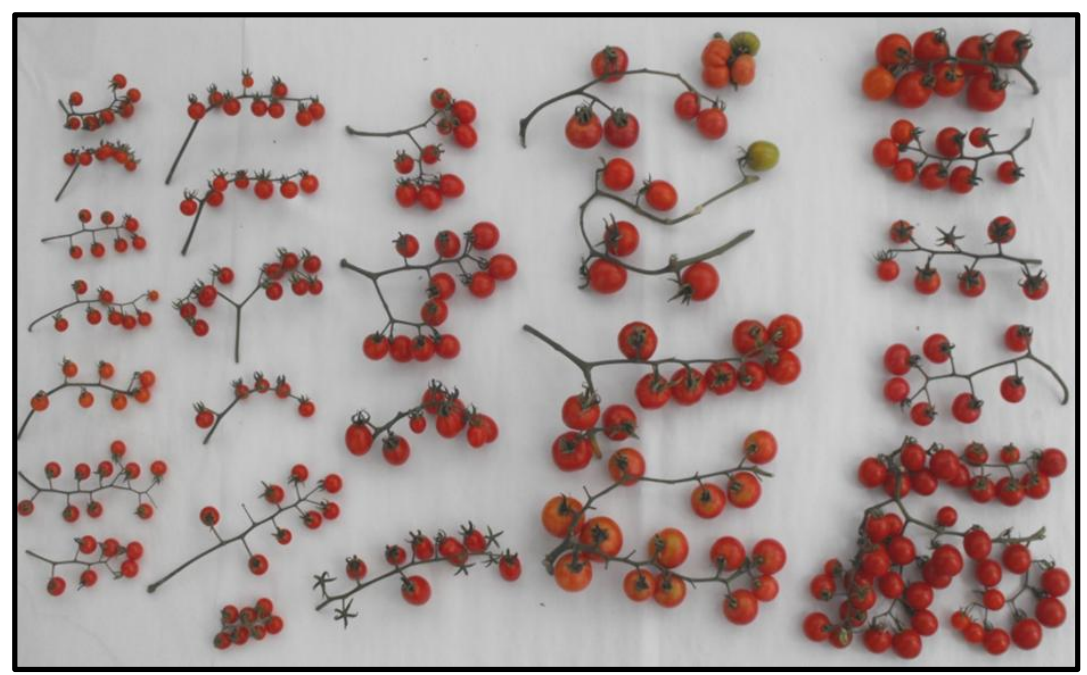

Figura 1. Frutos maduros de tomates silvestres del Estado de Oaxaca. 


\section{Variables respuesta}

Color. El color se evaluó mediante un fotocolorímetro portátil Mini Scan (HunterLab, modelo MS/B-200S®, USA), con lecturas en la escala CIE de $L^{*}, a^{*} y b^{*}$, con las que se calculó la relación $a^{*} / b^{*}$.

Composición química y nutricional. Los sólidos solubles, en jugo y pulpa, se cuantificaron con un refractómetro (Atago modelo 3T ${ }^{\circledR}$, Japan) y se expresaron en ${ }^{\circ}$ Brix (método 932.14 de la AOAC, 1990). Para el análisis del jugo se tomó una porción líquida antes del molido. El pH, la acidez titulable (\% de ácido cítrico) y el porcentaje de azúcares reductores se determinaron por los métodos 981.12, 942.15 y 923.09, respectivamente, de la AOAC (1990). La cuantificación del contenido de licopeno, en base húmeda y seca, se hizo con el método propuesto por Davis et al. (2006), mediante un espectrofotómetro Shimadzu, modelo UV-1601® (Japan), con lecturas a $503 \mathrm{~nm}$ y con base en una curva ajustada basada en un estándar de licopeno (90 \% de jugo puro de tomate, Sigma ${ }^{\circledR}$ ). El contenido de vitamina C (ácido ascórbico total) se determinó por el método de Durust et al. (1997), con lecturas en el mismo espectrofotómetro a una longitud de onda de $520 \mathrm{~nm}$, y basados en una curva de calibración con el estándar L-ácido ascórbico (99 \% de pureza, Sigma $\left.{ }^{\circledR}\right)$. Todas las determinaciones se hicieron por triplicado en cada parcela experimental.

\section{Análisis estadístico}

Se realizó un análisis de componentes principales por el método de la matriz de correlaciones y uno de conglomerados de agrupación jerárquica por el método de Ward; en ambos casos se utilizaron las variables estándarizadas. La definición de grupos o punto de corte del dendrograma se basó en la significancia del valor de t $(\mathrm{P} \leq 0.05)$, con ayuda del programa Clustan Graphics (Clustan ${ }^{\circledR}$; Clustan Graphics Version 5.22, Dec. 2001. Clustan Ltd Edinburgh, Scotland). Posteriormente, con base en la conducción del experimento en invernadero, se hizo un análisis de varianza con el modelo lineal de bloques completos al azar.

Cuando hubo diferencias entre grupos y colectas anidadas en grupos, se hizo una comparación de medias por el método de Tukey $(\mathrm{P} \leq 0.05)$. Para determinar la relación de las características químicas y nutricionales con las evaluaciones de color, se hizo un análisis de correlación simple de Pearson $(\mathrm{P} \leq 0.01)$.

\section{RESULTADOS Y DISCUSIÓN}

Los tres primeros componentes principales explicaron $82.3 \%$ de la variación total. El primer componente principal (CP1) estuvo determinado, en mayor grado, por el contenido de licopeno en base húmeda, sólidos solubles de jugo y pulpa, variaciones en color rojo (a* positivo) en el fruto y azúcares reductores; el CP2 por licopeno en base seca, luminosidad $\left(\mathrm{L}^{*}\right)$, color amarillo ( $\mathrm{b}^{*}$ positivo) $\mathrm{y}$ acidez titulable; y el $\mathrm{CP} 3$ por $\mathrm{pH}$ y licopeno en base seca. Con el análisis de conglomerados, las 17 colectas se clasificaron en tres grupos diferentes $(\mathrm{t}=5.11, \mathrm{gl}=15$, $P \leq 0.05)$, que fueron graficados en el plano determinado por los dos primeros componentes principales (Figura 2).

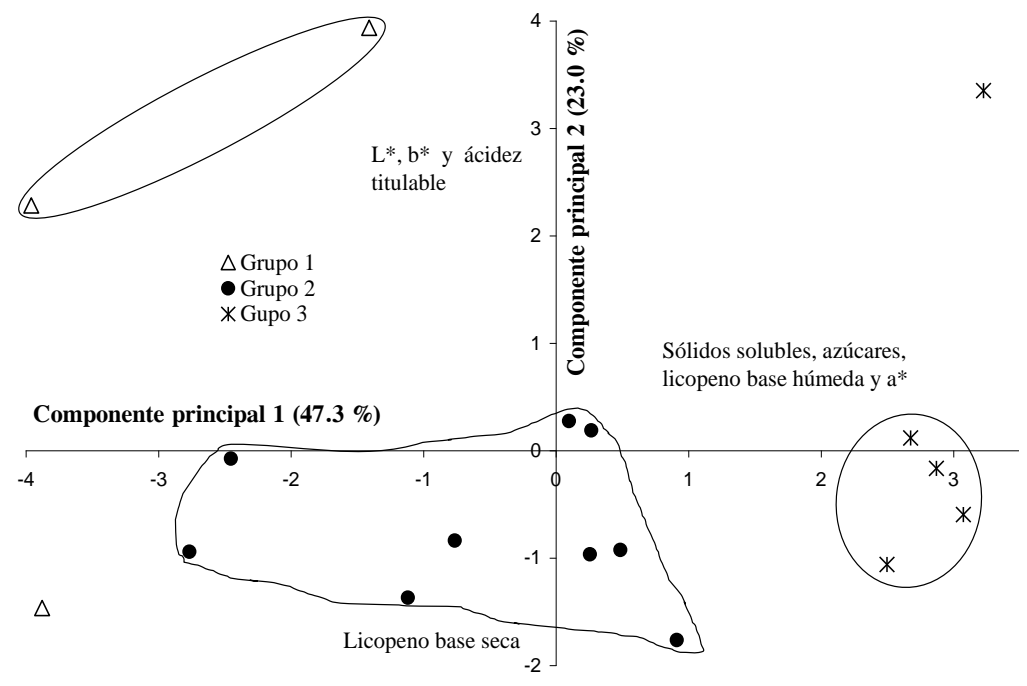

(a)

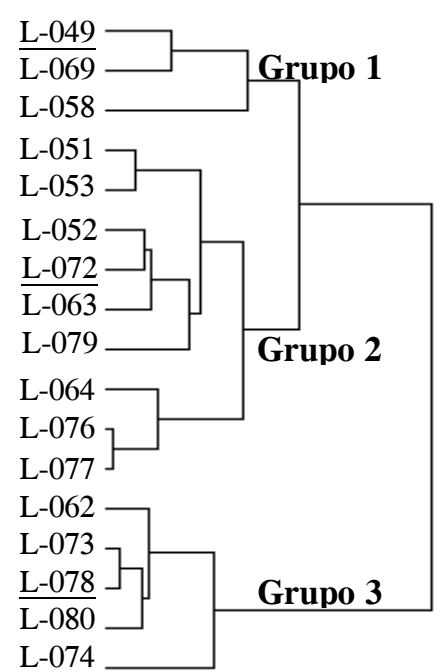

(b)

Figura 2. Dispersión de 17 colectas de tomate nativas de Oaxaca, en función de los dos primeros componentes principales (a); y su agrupamiento en el dendrograma (b), con base en 12 variables evaluadas. 
Las colectas evaluadas presentaron tres patrones generales de comportamiento (tres grupos), de acuerdo con el análisis de componentes principales y de conglomerados. El Grupo 1 fue el más disperso y se asoció con valores altos de $\mathrm{L}^{*}(40.3$ y 40.4$)$ y b* (58.3); el Grupo 2 incluyó a nueve colectas y se asoció con bajos valores de $\mathrm{L}^{*}(32.5$ a 38.7$)$, de $\mathrm{b}^{*}(34.0$ a 51.1$)$ y de acidez titulable (0.32 a $0.73 \%$ de ácido cítrico); y el Grupo 3 estuvo relacionado con los mayores contenidos de sólidos solubles ( 7.8 a $8.9^{\circ}$ Brix en jugo y de 8.4 a 9.3 ${ }^{\circ}$ Brix en pulpa).

La descripción y agrupamiento de las colectas evaluadas mediante los análisis multivariados, indicaron que es posible determinar diferentes patrones de variación en $L$. esculentum var. cerasiforme de Oaxaca, con base en los contenidos de licopeno, sólidos solubles, azúcares reductores, así como en las variaciones de color en el fruto. Las muestras originarias de Ayotzintepec (L-049), región de Tuxtepec; Teotitlán de Flores Magón (L-069), región de la Cañada; y Santa María Guenagati (L-058), región del Istmo (Grupo 1), contrastaron con las de San Blas Atempa (L-062, L-073 y L-078) región del Istmo, de Nejapa de Madero (L-080), Sierra Sur, y de Santiago Nuyoó (L-074), Mixteca (Grupo 3), (Figura 2).

Licopeno. Los valores más altos de licopeno en base húmeda correspondieron a las colectas L-063 del Grupo 2, y a L-073 y L-078 del Grupo 3, con 26.8, 28.2 y 26.3 $\mathrm{mg} 100 \mathrm{~g}^{-1}$, respectivamente, y difieren $(\mathrm{P} \leq 0.05)$ de las colectas que integran el Grupo 1 (L-049, L-058 y L-069). Este mismo patrón se repitió en el contenido de licopeno en base seca, y los valores máximos correspondieron a los materiales L-052, L-063 y L-073 (343.9 a $368.1 \mathrm{mg} 100$ $\left.\mathrm{g}^{-1}\right)$. Así, L-063 y L-073 mostraron mayor consistencia en mayor contenido de licopeno, tanto en base húmeda como seca (Cuadro 1).

En este trabajo la variación de licopeno en base seca fue de 116.6 a $368 \mathrm{mg} 100 \mathrm{~g}^{-1}$ en tomatillos de Oaxaca, y contrastó con la variación reportada por Juárez-López et al. (2009) de 33.4 a $51.9 \mathrm{mg} 100 \mathrm{~g}^{-1}$, en materiales de Guerrero y Puebla, aunque las diferencias entre ambos trabajos pueden ser producto de variaciones en las técnicas y estándares de licopeno utilizados. En este trabajo se hicieron las lecturas en el refractómetro a 503 nm y Juárez-López et al. (2009) las hicieron a $472 \mathrm{~nm}$. No obstante las diferencias en la técnica de determinación, no se descarta que haya diferencias genotípicas entre los acervos de Oaxaca, Puebla y Guerrero. Por otro lado, la variación aquí evaluada se encontró dentro del rango reportado por Guil-Guerrero y Rebolloso-Fuentes (2009), entre 28 y $350 \mathrm{mg} 100 \mathrm{~g}^{-1}$ para dos tomates tipo "Cherry" de Almería, España. En este mismo sentido, Raffo et al. (2002) estimaron un promedio de $141 \mathrm{mg} 100 \mathrm{~g}^{-1}$ para la variedad "Cherry" 'Naomi F1' en Sicilia, Italia. Esto indica cierto potencial de las colectas evaluadas, aun cuando se requiera una evaluación más cuidadosa para identificar el efecto genotípico.

Vitamina C. Entre colectas el ácido ascórbico varió de 6.1 a $16.1 \mathrm{mg} 100 \mathrm{~g}^{-1}$, y en los Grupos el 3 presentó el promedio $(\mathrm{P} \leq 0.05)$ más alto con $13.7 \mathrm{mg} 100 \mathrm{~g}^{-1}$. Las colectas sobresalientes fueron L-076, L-077, L-078 y L079, todas ellas de San Blas Atempa de la región del Istmo. Los valores más bajos correspondieron a las colectas que integran el Grupo 1, con un promedio de 8.6 $\mathrm{mg} 100 \mathrm{~g}^{-1}$ (Cuadro 1). Los valores de vitamina $\mathrm{C}$ aquí presentados son inferiores a los de Juárez-López et al. (2009), quienes reportaron variación desde 37 a $65.6 \mathrm{mg}$ $100 \mathrm{~g}^{-1}$ en colectas de Puebla y Guerrero, pero muy semejantes a los de Slimestad y Verheul (2005) y de Raffo et al. (2002), entre 5.6 y $20.0 \mathrm{mg} 100 \mathrm{~g}^{-1}$ en peso fresco en tomate tipo "Cherry". Aun con las diferencias o semejanzas en patrones de variación, entre éste y otros trabajos, es necesario destacar que los tomatillos de San Blas Atempa son muy populares en los mercados locales de Oaxaca; por ello, como algunas colectas son promisorias en contenido de vitamina $\mathrm{C}$, conviene documentar con mayor precisión la variación genética del tomatillo en esta región, a fin de promover la conservación y aprovechamiento de esos acervos genéticos.

Sólidos solubles totales. El contenido de sólidos solubles totales en jugo varió de 3.8 (L-058) a $8.9^{\circ}$ Brix (L-074) y en pulpa de 4.5 (L-058) a 9.3 (L-062) ${ }^{\circ}$ Brix. Además de las diferencias entre colectas, hubo diferencias $(\mathrm{P} \leq 0.05)$ entre grupos, con valores de 4.7, 5.5, y 8.3 ${ }^{\circ}$ Brix en jugo y de 5.2, 6.0 y $8.9^{\circ}$ Brix en pulpa, para los Grupos 1, 2, y 3, respectivamente (Cuadro 1). Las colectas L-062 de San Blas Atempa, de la región del Istmo y L-074 de Santiago Nuyoó de la región Mixteca, sobresalieron en sólidos solubles. Los resultados cubren parte de la variación reportada por Juárez-López et al. (2009), entre 5.8 y $8.0^{\circ}$ Brix, y ambos trabajos se pueden considerar complementarios. En este sentido, al comparar la variación de sólidos solubles entre los tomates tipos "Cherry", de este trabajo, junto con los evaluados por Juárez-López et al. (2009), y la variación estimada por García et al. (2009), (5.43 a $5.62^{\circ}$ Brix) para la variedad el tipo Bola 'EF 163', se deduce que, aun con las diferencias intrínsecas entre evaluaciones, es factible encontrar mayores concentraciones de azúcares en los tipo "Cherry" que en otros tipos, como concluyeron George $e t$ al. (2004). 
Cuadro 1. Características físicas, químicas y nutricionales de 17 colectas de tomate silvestre de Oaxaca, México. Ciclo primavera-verano 2009.

\begin{tabular}{|c|c|c|c|c|c|c|c|c|c|c|c|c|}
\hline Colecta & LBH & LBS & $\mathrm{VC}$ & SSJ & SSP & $\mathrm{pH}$ & AR & AT & $\mathrm{L}^{*}$ & $a^{*}$ & $\mathrm{~b}^{*}$ & $\mathrm{a}^{*} / \mathrm{b}^{*}$ \\
\hline L-049 & $7.1 \mathrm{fg}$ & $146.1 \mathrm{kl}$ & $6.6 \mathrm{~h}$ & $4.5 \mathrm{~h}$ & $5.0 \mathrm{j}$ & $4.22 \mathrm{~b}$ & $2.7 \mathrm{i}$ & $0.45 \mathrm{j}$ & $40.4 \mathrm{a}$ & $20.5 \mathrm{de}$ & $45.1 \mathrm{bcd}$ & $0.46 \mathrm{~d}$ \\
\hline L-058 & $9.1 \mathrm{f}$ & $195.6 \mathrm{ij}$ & $8.2 \mathrm{~g}$ & $3.8 \mathrm{i}$ & 4.51 & 3.631 & $2.0 \mathrm{j}$ & $0.59 \mathrm{f}$ & $34.6 \mathrm{bcd}$ & $18.6 \mathrm{e}$ & $28.8 \mathrm{f}$ & $0.65 \mathrm{abcd}$ \\
\hline L-069 & $7.0 \mathrm{~g}$ & 116.61 & $10.9 \mathrm{e}$ & $5.9 \mathrm{e}$ & $6.2 \mathrm{~g}$ & $4.17 \mathrm{~cd}$ & $2.8 \mathrm{hi}$ & $0.84 \mathrm{c}$ & $40.3 \mathrm{a}$ & $30.3 \mathrm{bc}$ & $58.3 \mathrm{a}$ & $0.52 \mathrm{~cd}$ \\
\hline Grupo 1 & $7.8 \mathrm{C}$ & $152.7 \mathrm{~B}$ & 8.6 C & $4.7 \mathrm{C}$ & $5.2 \mathrm{C}$ & $4.01 \mathrm{~B}$ & $2.5 \mathrm{C}$ & $0.52 \mathrm{C}$ & $38.4 \mathrm{~A}$ & $23.1 \mathrm{C}$ & $44.1 \mathrm{~A}$ & $0.54 \mathrm{C}$ \\
\hline L-051 & $12.7 \mathrm{e}$ & $266.3 \mathrm{def}$ & $6.1 \mathrm{~h}$ & $4.3 \mathrm{~h}$ & $4.8 \mathrm{k}$ & $4.30 \mathrm{a}$ & $2.8 \mathrm{~h}$ & 0.321 & $35.9 \mathrm{abcd}$ & $28.5 \mathrm{bcd}$ & $43.4 \mathrm{cde}$ & $0.65 \mathrm{abcd}$ \\
\hline L-052 & $19.2 \mathrm{~b}$ & $361.8 \mathrm{a}$ & $6.5 \mathrm{~h}$ & $4.9 \mathrm{~g}$ & $5.6 \mathrm{~h}$ & $4.17 \mathrm{bcd}$ & $3.2 \mathrm{~g}$ & $0.43 \mathrm{j}$ & $35.0 \mathrm{bcd}$ & $29.5 \mathrm{bcd}$ & 41.3 cde & $0.72 a b c$ \\
\hline L-053 & $12.6 \mathrm{e}$ & $252.1 \mathrm{efg}$ & $8.4 \mathrm{~g}$ & $4.2 \mathrm{~h}$ & $5.1 \mathrm{j}$ & $4.20 \mathrm{bc}$ & $2.8 \mathrm{~h}$ & $0.37 \mathrm{k}$ & $35.3 \mathrm{bcd}$ & $22.6 \mathrm{bcd}$ & 34.0 ef & $0.67 \mathrm{abc}$ \\
\hline L-063 & $26.8 \mathrm{a}$ & $368.1 \mathrm{a}$ & $9.9 \mathrm{f}$ & $7.1 \mathrm{c}$ & $6.9 \mathrm{e}$ & $4.07 \mathrm{fg}$ & $4.0 \mathrm{de}$ & $0.56 \mathrm{~g}$ & $33.7 \mathrm{bcd}$ & $30.3 \mathrm{bc}$ & 42.6 cde & $0.71 \mathrm{abc}$ \\
\hline L-064 & $16.2 \mathrm{~d}$ & $216.9 \mathrm{hi}$ & $9.8 \mathrm{f}$ & $6.4 \mathrm{~d}$ & $7.1 \mathrm{~d}$ & 4.04 ghi & $4.1 \mathrm{~d}$ & $0.64 \mathrm{e}$ & $33.3 \mathrm{~cd}$ & $32.4 \mathrm{ab}$ & $51.1 \mathrm{abc}$ & $0.63 \mathrm{bcd}$ \\
\hline L-072 & $18.2 \mathrm{~cd}$ & $290.7 \mathrm{~cd}$ & $12.8 \mathrm{~d}$ & $5.3 \mathrm{f}$ & $5.6 \mathrm{~h}$ & $4.02 \mathrm{ij}$ & $3.1 \mathrm{~g}$ & $0.54 \mathrm{~h}$ & $34.4 \mathrm{bcd}$ & $29.2 \mathrm{bcd}$ & 43.5 bcde & $0.67 \mathrm{abc}$ \\
\hline L-076 & $17.6 \mathrm{~cd}$ & 230.0 ghi & $16.1 \mathrm{a}$ & $6.4 \mathrm{~d}$ & $6.9 \mathrm{e}$ & $3.96 \mathrm{j}$ & $4.5 \mathrm{bc}$ & $0.51 \mathrm{i}$ & $32.9 \mathrm{~cd}$ & $29.7 \mathrm{bcd}$ & 42.4 cde & $0.70 \mathrm{abc}$ \\
\hline L-077 & $18.8 \mathrm{c}$ & $231.7 \mathrm{fgh}$ & $15.9 \mathrm{a}$ & $6.4 \mathrm{~d}$ & $6.6 \mathrm{f}$ & $3.88 \mathrm{k}$ & 3.9 ef & $0.57 \mathrm{fg}$ & $32.5 \mathrm{~d}$ & $29.4 \mathrm{bcd}$ & 43.4 cde & $0.68 \mathrm{abc}$ \\
\hline L-079 & $18.0 \mathrm{~cd}$ & $295.4 \mathrm{~cd}$ & $16.1 \mathrm{a}$ & $5.1 \mathrm{fg}$ & $5.2 \mathrm{i}$ & $4.10 \mathrm{ef}$ & $2.2 \mathrm{j}$ & $0.73 \mathrm{~d}$ & $38.7 \mathrm{ab}$ & $37.2 \mathrm{ab}$ & 44.2 bcde & $0.85 \mathrm{a}$ \\
\hline Grupo 2 & $17.8 \mathrm{~B}$ & $279.2 \mathrm{~A}$ & $11.3 \mathrm{~B}$ & $5.5 \mathrm{~B}$ & $6.0 \mathrm{~B}$ & $4.1 \mathrm{~A}$ & $3.4 \mathrm{~B}$ & $0.62 \mathrm{~B}$ & $34.6 \mathrm{~B}$ & $29.8 \mathrm{~B}$ & 42.9 A & $0.70 \mathrm{~B}$ \\
\hline L-062 & $22.6 \mathrm{~b}$ & $245.0 \mathrm{efgh}$ & $10.0 \mathrm{f}$ & $8.7 \mathrm{a}$ & $9.3 \mathrm{a}$ & $4.00 \mathrm{ij}$ & $4.4 \mathrm{c}$ & $1.07 \mathrm{~b}$ & $36.2 \mathrm{abcd}$ & $34.8 \mathrm{ab}$ & $43.4 \mathrm{cde}$ & $0.81 \mathrm{ab}$ \\
\hline L-073 & $28.2 \mathrm{a}$ & $343.9 \mathrm{ab}$ & $12.4 \mathrm{~d}$ & $8.2 \mathrm{~b}$ & $8.7 \mathrm{~b}$ & $4.14 \mathrm{de}$ & $4.5 \mathrm{bc}$ & $0.84 \mathrm{c}$ & $36.4 \mathrm{abcd}$ & $36.9 \mathrm{ab}$ & $46.3 \mathrm{bcd}$ & $0.80 \mathrm{ab}$ \\
\hline L-074 & $16.4 \mathrm{~d}$ & $173.8 \mathrm{jk}$ & $15.4 \mathrm{bc}$ & $8.9 \mathrm{a}$ & $9.1 \mathrm{a}$ & $4.06 \mathrm{fgh}$ & $3.8 \mathrm{f}$ & $1.45 \mathrm{a}$ & $40.7 \mathrm{a}$ & $40.4 \mathrm{a}$ & $54.0 \mathrm{ab}$ & $0.75 \mathrm{ab}$ \\
\hline L-078 & $26.3 \mathrm{a}$ & $313.1 \mathrm{bc}$ & $15.8 \mathrm{ab}$ & $7.8 \mathrm{~b}$ & $8.4 \mathrm{c}$ & $4.09 \mathrm{f}$ & $4.6 \mathrm{~b}$ & $0.84 \mathrm{c}$ & $37.7 \mathrm{abc}$ & $35.5 \mathrm{ab}$ & $45.4 \mathrm{bcd}$ & $0.78 \mathrm{ab}$ \\
\hline L-080 & $24.1 \mathrm{~b}$ & 280.2 cde & $15.1 \mathrm{c}$ & $7.9 \mathrm{~b}$ & $8.8 \mathrm{~b}$ & $4.14 \mathrm{de}$ & $4.9 \mathrm{a}$ & $0.84 \mathrm{c}$ & $35.2 \mathrm{bcd}$ & $30.7 \mathrm{bc}$ & $37.7 \mathrm{def}$ & $0.82 \mathrm{ab}$ \\
\hline Grupo 3 & $23.5 \mathrm{~A}$ & $272.0 \mathrm{~A}$ & $13.7 \mathrm{~A}$ & $8.3 \mathrm{~A}$ & $8.9 \mathrm{~A}$ & $4.1 \mathrm{~A}$ & $4.4 \mathrm{~A}$ & $1.00 \mathrm{~A}$ & $37.3 \mathrm{~A}$ & $35.7 \mathrm{~A}$ & $45.4 \mathrm{~A}$ & $0.79 \mathrm{~A}$ \\
\hline DSH-colecta & 2.07 & 34.80 & 0.55 & 0.36 & 0.15 & 0.05 & 0.13 & 0.03 & 4.93 & 9.47 & 10.57 & 0.21 \\
\hline Valor de $F$ & $282.8^{* *}$ & $120.2^{* *}$ & $1273.8^{* *}$ & $597.3^{* *}$ & $3144.9 * *$ & $290.7 * *$ & $1416.9 * *$ & $3402.7 * *$ & $8.19 * *$ & $10.7 * *$ & $11.8^{* *}$ & $7.0^{* *}$ \\
\hline CV (\%) & 3.82 & 4.46 & 1.55 & 1.90 & 0.75 & 0.38 & 1.16 & 1.23 & 4.46 & 10.19 & 7.88 & 9.62 \\
\hline
\end{tabular}

Medias con letras iguales, en la misma columna, no son estadísticamente diferentes (Tukey, 0.05 ); letras mayúsculas indican diferencia entre grupos. $* *=$ significativo a $\mathrm{P}<0.01 . \mathrm{CV}=$ coeficiente de variación; LBH y LBS = licopeno base húmeda y seca $\left(\mathrm{mg} / 100 \mathrm{~g}\right.$ de muestra); VC = vitamina $\mathrm{C}\left(\mathrm{mg} / 100 \mathrm{~g}\right.$ de muestra); SSJ y SSP = sólidos solubles en jugo y pulpa $\left({ }^{\circ} \mathrm{Brix}\right) ; \mathrm{AR}=\mathrm{azúcares}$ reductores $(\%)$; AT $=$ acidez titulable (\% de ácido cítrico). $\mathrm{L}^{*}=$ brillantez o luminosidad $\left(0\right.$, blanco a 100 , negro), $\mathrm{a}^{*}=$ variación de verde $(-)$ a rojo $(+)$ y $\mathrm{b}^{*}=$ variación de azul $(-)$ a amarillo (+). 
Sólidos solubles totales. El contenido de sólidos solubles totales en jugo varió de 3.8 (L-058) a $8.9^{\circ}$ Brix (L-074) y en pulpa de 4.5 (L-058) a 9.3 (L-062) ${ }^{\circ}$ Brix. Además de las diferencias entre colectas, hubo diferencias $(\mathrm{P} \leq 0.05)$ entre grupos, con valores de 4.7, 5.5, y 8.3 ${ }^{\circ}$ Brix en jugo y de 5.2, 6.0 y $8.9^{\circ}$ Brix en pulpa, para los Grupos 1, 2, y 3, respectivamente (Cuadro 1). Las colectas L-062 de San Blas Atempa, de la región del Istmo y L-074 de Santiago Nuyoó de la región Mixteca, sobresalieron en sólidos solubles. Los resultados cubren parte de la variación reportada por Juárez-López et al. (2009), entre 5.8 y $8.0^{\circ}$ Brix, y ambos trabajos se pueden considerar complementarios. En este sentido, al comparar la variación de sólidos solubles entre los tomates tipos "Cherry", de este trabajo, junto con los evaluados por Juárez-López et al. (2009), y la variación estimada por García et al. (2009), (5.43 a $5.62{ }^{\circ}$ Brix) para la variedad el tipo Bola 'EF 163', se deduce que, aun con las diferencias intrínsecas entre evaluaciones, es factible encontrar mayores concentraciones de azúcares en los tipo "Cherry" que en otros tipos, como concluyeron George et al. (2004).

pH. Se presentaron diferencias $(\mathrm{P} \leq 0.05)$ entre colectas, con una variación de 3.63 (L-058) a 4.3 (L051). Asimismo, hubo diferencia $(\mathrm{P} \leq 0.05)$ entre grupos, con 4.0 en el Grupo 1 y 4.1 para los Grupos 2 y 3 . En general, las colectas presentaron variación reducida $(\mathrm{CV}$ $=0.38 \%)$ y sólo las colectas L-058, L-076 y L-077, presentaron valores de $\mathrm{pH}$ menores a 4.0 (Cuadro 1).

Azúcares reductores. El porcentaje de azúcares reductores en los frutos varió de 2.0 (L-058) a $4.9 \%$ (L080) entre colectas, y entre grupos de 2.5 (Grupo 1) a 4.4 (Grupo 3). Las colectas L-073 y L-78 de San Blas Atempa y L-080 de Nejapa de Madero, ambas de la región del Istmo, fueron las más sobresalientes con valores de 4.5, 4.6 y $4.9 \%$, respectivamente (Cuadro 1 ).

Acidez titulable. Se encontraron diferencias $(\mathrm{P} \leq 0.05)$ entre grupos y entre colectas para la acidez titulable, expresada como porcentaje de ácido cítrico, que varió de 0.32 (L-051) a $1.45(\mathrm{~L}-074)$. El Grupo 3 presentó el mayor porcentaje $(1.0 \%)$, seguido del Grupo $2(0.62 \%)$ y finalmente del Grupo $1(0.52 \%) \quad(\mathrm{P} \leq 0.05)$. Nuevamente las colectas L-062 de San Blas y L-074 de Santiago Nuyoó presentaron los valores más altos (P $\leq$ 0.05 ), 1.07 y $1.45 \%$, respectivamente (Cuadro 1). Juárez-López et al. (2009) reportaron una variación de 0.5 a $1.1 \%$ de ácido cítrico en tomates tipo "Cherry" de Puebla y Guerrero.

George et al. (2004), en tres variedades de tomate tipo "Cherry" cultivada en Nueva Delhi, India, reportaron una variación de 0.52 a $0.7 \%$, y García et al. (2009) una variación de 0.46 a $0.51 \%$ en la variedad de tomate tipo Bola 'EF163'. Los resultados sugieren la presencia de cierto potencial en ácido cítrico en $L$. esculentum var. cerasiforme distribuido en México.

Evaluaciones de color. Los valores de color, expresados en $L^{*}, a^{*}, b^{*}$ y $a^{*} / b^{*}$, variaron $(P \leq 0.05)$ entre las colectas, de 32.5 a 40.7 , de 18.6 a 40.4 , de 28.8 a 58.3 y 0.52 a 0.85 , respectivamente. En brillo o luminosidad de la superficie del fruto $\left(\mathrm{L}^{*}\right)$ y en las variaciones de rojo $\left(a^{*}\right)$ y amarillo $\left(b^{*}\right)$, sobresalieron el Grupo 3, y entre colectas destacó consistentemente L-074, originaria de Santiago Nuyoó de la región Mixteca. Los valores estimados para la relación $\mathrm{a}^{*} / \mathrm{b}^{*}$ sugieren mayor variación entre grupos $(0.54$ a 0.79$)$ y menor variación dentro de grupos. Las magnitudes fueron de 0.46 a 0.65 para el grupo Grupo 1, de 0.63 a 0.85 en el Grupo 2 y de 0.75 a 0.82 en el grupo Grupo 3. Estos resultados indican que la evaluación del color, incluida la relación $\mathrm{a}^{*} / \mathrm{b}^{*}$, no permitió precisar diferencias entre colectas, pero permite hacer una estimación objetiva de la percepción del consumidor, si se consideran evaluaciones en todos los estados de maduración.

Las correlaciones entre las coordenadas cromáticas de color $\left(\mathrm{L}^{*}, \mathrm{a}^{*}, \mathrm{~b}^{*}\right.$ y $\left.\mathrm{a} * / \mathrm{b}^{*}\right)$ y las variables químicas y nutricionales aquí evaluadas, presentaron relaciones diferentes $(\mathrm{P} \leq 0.01)$. Las variaciones del color rojo $\left(\mathrm{a}^{*}\right)$ presentaron una correlación positiva y significativa $(\mathrm{r}>$ $0.4, \mathrm{P}<0.01)$ con licopeno en base húmeda, vitamina $\mathrm{C}$, sólidos solubles, azúcares y acidez titulable. La relación $\mathrm{a}^{*} / \mathrm{b}^{*} \mathrm{o}$ índice de color presentó una correlación positiva con todos los caracteres, excepto con $\mathrm{pH}$ (Cuadro 2). Esto sugiere que, en trabajos con mayor número de colectas, se ponga atención en las relaciones entre las variables químicas y nutricionales y en los estimadores de color $\mathrm{a}^{*} \mathrm{y} \mathrm{a}^{*} / \mathrm{b}^{*}$.

Cuadro 2. Coeficientes de correlación de Pearson (r) entre características químicas y nutricionales, $y$ medidas del color de fruto en tomate silvestre de Oaxaca, México.

\begin{tabular}{|c|c|c|c|c|}
\hline \multirow{2}{*}{ Variable } & \multicolumn{4}{|c|}{ Coordenadas y relación cromática } \\
\hline & $\mathrm{L}^{*}$ & $\mathrm{a}^{*}$ & $\mathrm{~b}^{*}$ & $\mathrm{a} * / \mathrm{b}^{*}$ \\
\hline Licopeno base húmeda & -0.27 & $0.53 * *$ & -0.07 & $0.69 * *$ \\
\hline Licopeno base seca & -0.35 & 0.25 & -0.23 & $0.50 * *$ \\
\hline Vitamina $\mathrm{C}$ & $<0.01$ & $0.54 * *$ & 0.21 & $0.45^{* *}$ \\
\hline Sólidos solubles en jugo & 0.10 & $0.65^{* *}$ & 0.29 & $0.51 * *$ \\
\hline Sólidos solubles en pulpa & 0.08 & $0.60 * *$ & 0.23 & $0.51 * *$ \\
\hline $\mathrm{pH}$ & 0.37 & 0.18 & 0.37 & -0.13 \\
\hline Azúcares reductores & -0.25 & $0.45^{* *}$ & 0.11 & $0.43 * *$ \\
\hline Acidez titulable & $0.41 * *$ & $0.60 * *$ & 0.34 & $0.40 * *$ \\
\hline
\end{tabular}

**Significativo a nivel de $\mathrm{P}<0.01$ (t de Student). 


\section{CONCLUSIONES}

Los contenidos de licopeno, sólidos solubles, $\mathrm{pH}$, azúcares reductores y acidez titulable, y los parámetros de color, fueron las variables más importantes para describir y clasificar en tres grupos la variación evaluada en 17 colectas de tomate tipo "Cherry" de Oaxaca. Hubo diferencias $(P \leq 0.05)$ entre grupos y entre colectas para todas las variables. El Grupo 3, integrado por tres colectas de San Blas Atempa, región del Istmo, una de Nejapa de Madero, Sierra Sur y otra de Santiago Nuyoó, Mixteca, en todas las variables presentó los valores más altos. Las colectas L-063 y L-073, ambas de San Blas Atempa, sobresalieron en licopeno base húmeda, con 26.8 y $28.2 \mathrm{mg} 100 \mathrm{~g} \mathrm{~g}^{-1}$, y en base seca con 368.1 y $343.9 \mathrm{mg}$ $100 \mathrm{~g}^{-1}$, respectivamente. En vitamina $\mathrm{C}$ sobresalieron las colectas L-074 de Santiago Nuyoó, L-076, L-077, L-078 y L-079 de San Blas Atempa, y L-080 de Nejapa de Madero, Oaxaca, con más de $15 \mathrm{mg} 100 \mathrm{~g}^{-1}$. El índice de color $\left(\mathrm{a}^{*} / \mathrm{b}^{*}\right)$ correlacionó en forma positiva con todos los caracteres químicos y nutricionales, excepto con $\mathrm{pH}$.

\section{BIBLIOGRAFÍA}

Álvarez-Hernández J C, H Cortez-Madrigal, I García-Ruiz (2009) Exploración y caracterización de poblaciones silvestres de tomate (Solanaceae) en tres regiones de Michoacán, México. Polibotánica 28:139-159.

AOAC, Association of Official Analytical Chemists (1990) Official methods of analysis of the Association of Official Analytical Chemists. Vol. 2, 15th ed. Association of Official Analytical Chemists, Washington DC, USA. pp:685-1298.

Córdoba L, J C Molina (2006) Conservación ex situ. In: Recursos Fitogenéticos de México para la Alimentación y la Agricultura: Informe Nacional 2006. J C Molina, L Córdoba (eds). Secretaría de Agricultura, Ganadería, Desarrollo Rural, Pesca y Alimentación y Sociedad Mexicana de Fitogenética, A.C. Chapingo, México. pp:59-100.

Davis A R, W W Fish, P Perkins-Veazie (2006) A rapid spectrophotometric method for analyzing lycopene content in tomato and tomato products. Postharv. Biol. Technol. 28:425430 .
Durust N, D Sumengen, Y Durust (1997) Ascorbic acid and element contents of foods of Trabzon (Turkey). J. Agric. Food Chem. 45:2085-2087.

García M L, V Martínez, A N Avendaño, M C Padilla, H Izquierdo (2009) Acción de oligosacáridos en el rendimiento y calidad de tomate. Rev. Fitotec. Mex. 32:295-301.

George B, C Kaur, D S Khurdiya, H C Kapoor (2004) Antioxidants in tomato as a function of genotype. Food Chem. 84:45-51.

Guil-Guerrero J L, M M Rebolloso-Fuentes (2009) Nutrient composition and antioxidant activity of eight tomato (Lycopersicon esculentum) varieties. J. Food Comp. Anal. 22:123-129.

Jenkins J A (1948) The origin of the cultivated tomato. Econ. Bot. 2:379-392.

Juárez-López P, R Castro-Brindis, T Colinas-León, P RamírezVallejo, M Sandoval-Villa, D W Reed, L Cisneros-Zevallos, S King (2009) Evaluación de calidad de frutos de siete genotipos nativos de jitomate (Lycopersicon esculentum var. cerasiforme). Rev. Chapingo S. Hort. 15:5-9.

Kavanaugh C J, P R Trumbo, K C Ellwood (2007) The U.S. food and drug administration's evidence-based review for qualified health claims: Tomatoes, lycopene, and cancer. J. Nat. Cancer Inst. 99:1074-1085.

Lépiz R, E Rodríguez (2006) Los recursos fitogenéticos de México. In: Recursos Fitogenéticos de México para la Alimentación y la Agricultura: Informe Nacional 2006. J C Molina, L Córdoba (eds). Secretaría de Agricultura, Ganadería, Desarrollo Rural, Pesca y Alimentación y Sociedad Mexicana de Fitogenética, A.C. Chapingo, México. pp:1-58.

Peralta I E, D M Spooner (2007) History, origin and early cultivation of tomato (Solanaceae). In: Genetic Improvement of Solanaceous Crop, Vol. 2: Tomato. M K Razdan, A K Mattoo (eds). Science Publishers. Enfield, New Hampshire, USA. pp:1-24.

Raffo A, C Lonardi, V Fogliano, P Ambrosino, M Salucci, L Gennaro, R Bugianesi, F Giuffrida, G Quaglia (2002) Nutritional value of cherry tomatoes (Lycopersicon esculentum cv. Naomi F1) harvested at different ripening stages. J. Agric. Food Chem. 50:6550-6556.

Rick C M, J F Fobes (1975) Allozyme variation in the cultivated tomato and closely related species. Bull. Torrey Bot. Club 102:376-384.

Singh P, G K Goyal (2008) Dietary lycopene: its properties and anticancirnogenic effects. Compreh. Rev. Food Sci. Food Safety 7:255-270.

Slimestad R, M J Verheul (2005) Seasonal variations in the level of plant constituents in greenhouse production of cherry tomatoes. J. Agric. Food Chem. 53:3114-3119. 